\title{
Increasing trend of fluconazole-non-susceptible Cryptococcus neoformans in patients with invasive cryptococcosis: a 12-year longitudinal study
}

\author{
Yi-Chun Chen ${ }^{1}$, Tzu-Yao Chang ${ }^{1}$, Jien-Wei Liu ${ }^{1,2}$, Fang-Ju Chen ${ }^{1}$, Chun-Chih Chien ${ }^{3}$, Chen-Hsiang Lee ${ }^{1,2^{*}}$ \\ and Cheng-Hsien $\mathrm{Lu}^{2,4,5^{*}}$
}

\begin{abstract}
Background: This study aimed to investigate the rate of fluconazole-non-susceptible Cryptococcus neoformans in Southern Taiwan for the period 2001-2012 and analyze the risk factors for acquiring it among patients with invasive cryptococcosis.

Methods: All enrolled strains were isolated from blood or cerebrospinal fluid samples of the included patients. If a patient had multiple positive results for $C$. neoformans, only the first instance was enrolled. Susceptibility testing was performed using the Clinical and Laboratory Standards Institutes M27-A3 broth micro-dilution method. The MIC interpretative criteria for susceptibility to fluconazole were $\leq 8 \mu \mathrm{g} / \mathrm{ml}$. A total of 89 patients were included. Patients ( $n=59)$ infected by fluconazole-susceptible strains were compared with those $(n=30)$ infected by non-susceptible strains. The patients' demographic and clinical characteristics were analyzed.

Results: The rate of fluconazole-non-susceptible C. neoformans in the study period significantly increased over time $(p<0.001)$. The C. neoformans isolated in 2011-2012 (odds ratio: 10.68; $95 \%$ confidence interval: 2.87-39.74;

$p<0.001$ ) was an independent predictive factor for the acquisition of fluconazole-non-susceptible C. neoformans.

Conclusions: The rate of fluconazole-non-susceptible C. neoformans has significantly increased recently. Continuous and large-scale anti-fungal susceptibility tests for $C$. neoformans are warranted to confirm this trend.
\end{abstract}

Keywords: Cryptococcus neoformans, Invasive cryptococcosis, Susceptibility, Fluconazole, Risk factors

\section{Background}

Cryptococcus neoformans is an encapsulated yeast responsible for life-threatening infections [1]. Pharmacologic management usually consists of primary therapy with amphotericin B, with or without flucytosine, followed by maintenance therapy with fluconazole [2]. Pre-emptive fluconazole therapy for positive serum cryptococcal antigen in patients with human immunodeficiency virus (HIV) to prevent the development of cryptococcal meningitis is also regarded as cost effective for specific groups [3]. The widespread use of fluconazole for long-term suppressive therapy of cryptococcal

\footnotetext{
* Correspondence: lee900@adm.cgmh.org.tw; chlu99@ms44.url.com.tw 'Division of Infectious Diseases, Department of Internal Medicine, Kaohsiung Chang Gung Memorial Hospital, Kaohsiung, Taiwan

${ }^{2}$ Chang Gung University College of Medicine, 123 Ta-Pei RoadNiao-Sung District, Kaohsiung City 833, Taiwan

Full list of author information is available at the end of the article
}

infection has become a concern due to the development of fluconazole resistance $[4,5]$. Yet despite these concerns, in vitro susceptibility testing of $C$. neoformans isolates at the start of therapy is not routinely practiced [2]. Instead, it is reserved for patients with failed primary therapy, those with relapse, or those who develop cryptococcosis and have recent exposure to an antifungal drug [2].

In the guidelines of the Infectious Diseases Society of America (IDSA), primary resistance of C. neoformans to fluconazole is not a significant clinical problem, as noted in a previous study [6]. In a national surveillance in Taiwan in 2003, only three of 70 (4\%) C. neoformans clinical isolates had MICs of fluconazole at concentrations $\geq 16 \mu \mathrm{g} / \mathrm{ml}$ [7]. Another population-based surveillance in South Africa on C. neoformans isolates collected before 2008 still maintained low MIC values 
to fluconazole [8]. However, the development of microbial resistance is dynamic. The issue of using azoles and C. neoformans resistance has been described [9].

A previous study has found that the $\mathrm{MIC}_{90}$ of fluconazole against $C$. neoformans increased from 4 to $16 \mu \mathrm{g} / \mathrm{ml}$ in cerebrospinal fluid (CSF) specimens collected between 2001 and 2010 [10]. High rates of C. neoformans persistence and frequent relapses have sparked concern for the possible emergence of fluconazole resistance [11]. Increasing in vitro resistance to fluconazole in C. neoformans isolates has also been proposed [12]. A previous study has elucidated a correlation between fluconazole susceptibility and clinical outcome in patients with cryptococcal meningitis [10]. Thus, an updated surveillance of anti-fungal susceptibility of the clinical strain of C. neoformans is desirable to monitor the trend of fluconazole non-susceptible strains [13].

The current study aimed to evaluate the in vitro susceptibility of clinical C. neoformans isolates against fluconazole between 2001 and 2012 in Southern Taiwan. It also analyzed the risk factors for acquiring fluconazolenon-susceptible $C$. neoformans among patients with invasive cryptococcosis, which was defined as $C$. neoformans escape from the lungs and entering the bloodstream, thereby allowing central nervous system (CNS) dissemination [14].

\section{Methods}

\section{Study design}

C. neoformans isolated from patients with invasive cryptococcosis were collected. If the patient had more than one episode of invasive cryptococcosis, only the first episode was included. All enrolled clinical strains were isolated from blood or CSF samples of patients who were admitted to Kaohsiung Chang Gung Memorial Hospital (KCGMH) between January 2001 and December 2012. The KCGMH was a 2700-bed primary care and tertiary referral center in Southern Taiwan. If a patient had multiple positive results for C. neoformans, only the first isolate was enrolled for in vitro analysis.

This study followed previously published studies and included 46 clinical strains from CSF samples as described previously [10]. To determine the risk factors for acquiring fluconazole-non-susceptible C. neoformans, demographic and clinical information were retrieved from medical records retrospectively. The Chang Gung Memorial Hospital's Institutional Review Board approved the study [No. 102-3819B].

\section{Data collection and definition}

Data on clinical variables included age, sex, and underlying diseases (i.e., AIDS, diabetes mellitus, receiving hemodialysis, chronic kidney disease, liver cirrhosis, chronic lung disease, steroids usage, malignancy, hematologic malignancy, and autoimmune disease). The use of azoles was defined as the intake of fluconazole, itraconazole, voriconazole, or ketoconazole for more than $48 \mathrm{~h}$ within three months prior to the first episode of invasive cryptococcosis. Steroid use was defined as the intake of at least $10 \mathrm{mg}$ prednisolone or its equivalent per day for more than two months prior to the infection. The severity of illness at the time of CSF or blood sampling was assessed using the APACHE II scoring method [15] modified as 0 point given to the items $\mathrm{PaO}_{2}$ and $\mathrm{pH}$ if arterial blood gas analysis was not performed because of the absence of respiratory distress.

Sepsis was defined as a systemic response to infection, manifested by two or more of the following conditions: (1) temperature $>38{ }^{\circ} \mathrm{C}$ or $<36{ }^{\circ} \mathrm{C}$; (2) heart rate $>90$ beats per minute; (3) respiratory rate $>20$ breaths per minute or $\mathrm{PaCO}_{2}<32 \mathrm{~mm} \mathrm{Hg}$; and white blood cell count $>12,000$ cells $/ \mathrm{mm}^{3},<4,000$ cells $/ \mathrm{mm}^{3}$, or $>10 \%$ immature (band) forms [16]. Septic shock was diagnosed if there was refractory hypotension, signifying that intravenous fluid administration alone was insufficient to maintain adequate blood pressure [16]. The collected laboratory data was on leukocytes, hemoglobin, platelet count, percentage of neutrophils and lymphocytes, and presentation of high titers of CSF and serum cryptococcal antigen defined as more than 1:512 if these data were available.

\section{Fungal strain}

The processing of specimens and identification of isolates were performed by conventional methods using the Vitek Yeast Biochemical Card (BioMerieux, Marcy l'Etoile, France) [17]. The isolated strains were preserved at $-70{ }^{\circ} \mathrm{C}$ until the experiments.

\section{Isolation of genomic DNA and PCR amplification}

Each strain was grown on SDA plates at $35{ }^{\circ} \mathrm{C}$ for two days. Cells were collected and suspended in a TE buffer (100 mM Tris-HCl; pH 8.0, 1 mM EDTA) containing lyticase (Sigma, St. Louis, MO, USA). Glass beads (Sigma) were then added to the micro-tubes and the samples were incubated at $37{ }^{\circ} \mathrm{C}$ for $4 \mathrm{~h}$, and mixed in an end-over-end mixer to digest the cell walls. Genomic DNA was extracted from the cells using the High Pure PCR template preparation kit (Roche Applied Science, Mannheim, Germany) according to the manufacturer's instructions.

The serotype of $C$. neoformans was identified by multiplex PCR. Four primers for cloning laccase gene ( $L A C 1)$ and two for capsule gene (CAP64) were used [18]. The LAC1 differentiated serotypes $\mathrm{A}, \mathrm{D}, \mathrm{B}$ and $\mathrm{C}$ and CAP64 differentiated serotypes $\mathrm{D}$ and $\mathrm{AD}$. $\mathrm{CNa}-70-\mathrm{S}$ and $\mathrm{CNa}-$ 70-A primer pair (amplified a 695-bp DNA fragment 
from serotype A), $\mathrm{CNa}-29-\mathrm{S}$ and $\mathrm{CNa}-29-\mathrm{A}$ primer pair (amplified a 579-bp fragment from serotype A), CNa-29$\mathrm{S}$ and CNa-70-A primer pair (amplified a 666-bp or a 460-bp fragment from serotype A, 290-bp from serotype $\mathrm{B}$ or $\mathrm{C}$ ) and $\mathrm{CNb}-49-\mathrm{S}$ and $\mathrm{CNb}-49-\mathrm{A}$ primer pair (amplified a 448-bp fragment from serotype B or serotype C) were used [19]. The amplified products were separated by electrophoresis and stained with ethidium bromide. The DNA bands were extracted using a gel extraction kit (QIAquick, QIAGEN Sciences, Germantown, MD, USA) and sequenced directly using a BigDye Terminator Cycle-sequencing kit (ABI PRISM 310NT Genomic Analyzer, Perkin-Elmer Applied Biosystems, Foster, CA, USA).

\section{Fluconazole susceptibility testing}

Fluconazole (Pfizer, New York, NY, USA) susceptibilities were determined using the broth micro-dilution method according to the CLSI M27-A3 methodology [20]. Stock solutions were prepared in water and further diluted in RPMI 1640 medium (Sigma) buffered to a $\mathrm{pH}$ of 7 with $0.165 \mathrm{M}$ 3-( $N$-morpholino) propanesulfonic acid buffer (Sigma). Aliquots of each agent $(0.1 \mathrm{ml})$ at two-times the concentrations were dispensed into 96-well micro-dilution trays. The yeast inoculum was adjusted to a concentration of $10^{4} \mathrm{CFU} / \mathrm{mL}$ before being added to each well. The trays were incubated at $35{ }^{\circ} \mathrm{C}$. The final fluconazole concentrations ranged from 0.25 to $64 \mu \mathrm{g} / \mathrm{ml}$.

The MICs for fluconazole were the concentrations causing a $50 \%$ reduction in turbidity compared to the growth of the control at $72 \mathrm{~h}$. Candida krusei ATCC 6258 and Candida parapsilosis ATCC 22019 were used as quality controls. The interpretative criterion for susceptibility to fluconazole was $\leq 8 \mu \mathrm{g} / \mathrm{ml}$, as published by the CLSI [20]. The reproducibility of the in vitro results was assessed twice on two different days. The geometric mean fluconazole MIC was evaluated each year during the study period.

\section{Statistical analysis}

The annual fluconazole-non-susceptible rate of C. neoformans isolated from the patients was calculated. Chisquare test for a linear regression analysis was performed to determine the trends of fluconazole-non-susceptible rates of C. neoformans isolated from 2001 to 2012, while simple linear regression was used to estimate the trends of geometric mean fluconazole MIC during the study period. By univariate analysis, continuous variables were expressed as mean \pm standard deviation and values were compared by Student's $t$ test. Categorical variables, expressed as numbers and percentages, were compared by chi-square test or Fisher's exact test, as appropriate.

To identify risk factors for acquiring fluconazole-nonsusceptible $C$. neoformans, the patients were categorized as those with fluconazole-non-susceptible (Group 1) or with fluconazole-susceptible (Group 2) C. neoformans. Statistically significant variables in univariate analyses between these categories were entered into multivariate analysis using a logistic regression model. Statistical significance was set at a two-tailed $p<0.05$. Statistical analysis was conducted using the SPSS statistical analysis system (ver.21).

\section{Results}

During the study period, 93 isolates of Cryptococcus species were identified from blood $(n=48)$ and CSF $(n=45)$ samples. Eighty-nine isolates were C. neoformans var. grubii (serotype A) and 4 were C. gattii (serotype B). Of the 89 C. neoformans isolates, 30 (34 \%) were fluconazolenon-susceptible (MICs $\geq 16 \mu \mathrm{g} / \mathrm{ml}$ ). The 89 patients with invasive cryptococcosis were categorized as those who infected by fluconazole non-susceptible $C$. neoformans (Group 1, $n=30$ ) and those infected by fluconazole susceptible C. neoformans (Group 2, n=59). The annual rate of fluconazole non-susceptible $C$. neoformans isolated from 2001 to 2012 significantly increased over time $(p<0.001)$. The annual rate of fluconazole nonsusceptible C. neoformans was $0-33 \%$ in 2001-2006, $18-29 \%$ in 2007-2010, and 75-86 \% in 2011-2012 (Fig. 1). There is also a significantly increasing trend over time in the geometric mean fluconazole MIC $(p<0.01)$. Clinical strains cultured in 2011-2012 were specified to clarify the emergence of fluconazolenon-susceptible strains.

The demographic and clinical features and serotypes of $C$. neoformans strains between the two groups were compared (Table 1). Except for the lower proportion of liver cirrhosis in Group 1 (3 \% vs. $24 \%, p=0.02$ ), the demographic and clinical features were similar in the two groups. Patients in Group 1 had a predominated admission during 2011-2012 (50\% vs. $7 \%, p<0.001$ ), previous azole exposure ( $24 \%$ vs. $7 \%, p=0.04)$, and initial presentation as sepsis (70 \% vs. $46 \%, p=0.03$ ).

In multivariate analysis, patient admission in 20112012 (odds ratio, OR: 10.68; $95 \%$ confidence interval, CI: $2.87-39.74 ; p<0.001)$ was an independent predictive factor for acquiring fluconazole-non-susceptible C. neoformans (Table 2).

\section{Discussion}

The current study demonstrated that the rate of fluconazole non-susceptible C. neoformans from 2001 to 2012 significantly increased over time $(p<0.001)$. A high fluconazole-non-susceptible rate was especially recognized in the last two years (2011-2012). In the past, the development of secondary resistance to fluconazole during therapy was given more attention than primary resistance. During the 12-year study period, there were 89 


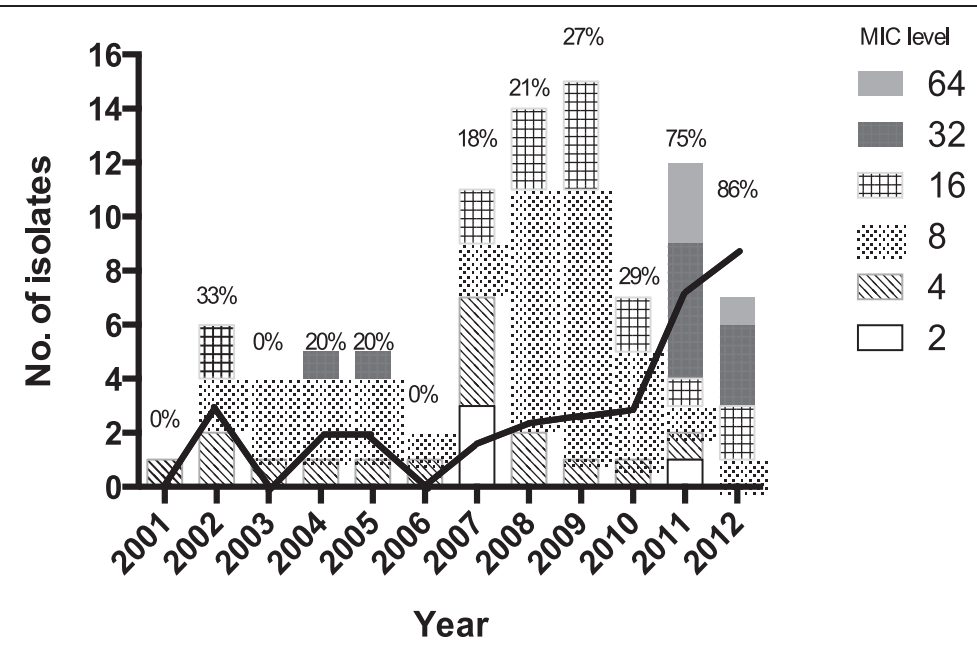

\begin{tabular}{|l|l|l|l|l|l|l|l|l|l|l|l|l|}
\hline Year & 2001 & 2002 & 2003 & 2004 & 2005 & 2006 & 2007 & 2008 & 2009 & 2010 & 2011 & 2012 \\
\hline Geometric & 4 & 8 & 6.7 & 9.2 & 9.2 & 5.7 & 4.8 & 8.4 & 9.2 & 8.8 & 21.4 & 23.8 \\
MIC $(\mu \mathrm{g} / \mathrm{ml})$ & & & & & & & & & & & & \\
\hline
\end{tabular}

Fig. 1 The annual rate (black line) of fluconazole non-susceptible (MICs $\geq 16 \mu \mathrm{g} / \mathrm{ml}$ ) Cryptococcus neoformans from 2001 to 2012 significantly increased over time $(p<0.001)$. There was also an increasing trend of geometric mean fluconazole MIC during the study period $(p<0.01)$

C. neoformans initial clinical isolates from patients with invasive cryptococcosis, including 30 (34 \%) nonsusceptible to fluconazole.

The rate of fluconazole non-susceptible C. neoformans in the present study is higher than those of previous studies $[6,7,21]$. Thus, non-susceptibility to fluconazole has become a growing problem. A global anti-fungal surveillance study conducted from 1997 to 2007 documents a progressive increase in resistance to fluconazole among C. neoformans isolates when results from the time periods 1997 to 2000 (7.3\%), 2001 to 2004 (10.9\%), and 2005 to 2007 (11.7\%) are compared [22]. The problem is especially true among isolates from the Asia-Pacific, Africa/Middle East, and Latin America compared to isolates from Europe or North America [22].

In Asia, most invasive cryptococcosis cases are caused by $C$. neoformans var. grubii [23, 24]. Similarly, $95.7 \%$ (89/93) of C. neoformans isolates from the present study belong to serotype A. One previous study has demonstrated that strains of serotype A are less susceptible to fluconazole than strains of serotype D [21]. Using microsatellite analysis, there is a different distribution of genotypes of $C$. neoformans var. grubii isolates in various countries in Asia, as well as a correlation of the microsatellite genotypes with the original source of the strain and resistance to anti-fungal agents [25]. Recently, a nationwide multi-center retrospective study in Taiwan has suggested that $C$. neoformans isolates with antifungal MIC higher than the epidemiologic cut-off values are rare (one of 203 isolates had fluconazole MIC levels $>8 \mu \mathrm{g} / \mathrm{mL}$ ) [26, 27]. In Spain, 58 C. neoformans clinical isolates were collected from 1990 to 2007 . Only 2 strains isolated from $\mathrm{HIV}$ patients were fluconazole MIC of $16 \mu \mathrm{g} / \mathrm{ml}$ (3.4 \%) [28]. Although the number of fluconazole non-susceptible $C$. neoformans is small according to these two recent studies, the clinical isolates have been obtained before 2010 [27, 28].

In the present study, more patients infected by fluconazole-non-susceptible $C$. neoformans strains experienced azole exposure recently than those infected by fluconazole-susceptible $C$. neoformans strains. The azole exposure in our study is not an independent risk factor for invasive cryptococcosis caused by fluconazolenon-susceptible $C$. neoformans under the multivariate analysis, but the exposure history of our patients may be underestimated because of retrospective study nature. One case series showed that $70 \%$ of patients with fluconazole-resistant cryptococcosis had history of prior exposure to fluconazole [29]. Therefore, the IDSA guidelines for the management of cryptococcal disease recommended in-vitro susceptibility testing should be reserved for patients had recent exposure to an antifungal drug [2]. 
Table 1 Risk factors for invasive cryptococcosis caused by fluconazole-non-susceptible C. neoformans

\begin{tabular}{|c|c|c|c|}
\hline Variables & Group 1, $n=30(\%)$ & Group 2, $n=59(\%)$ & $p$ \\
\hline Age & $53.8 \pm 17.9$ & $58.3 \pm 17.8$ & 0.27 \\
\hline Male: female & $21: 9$ & $37: 22$ & 0.50 \\
\hline Admissions 2011-2012 & $15(50)$ & $4(7)$ & $<0.001$ \\
\hline Azole exposure ${ }^{a}$ & $7(24)$ & $4(7)$ & 0.04 \\
\hline \multicolumn{4}{|l|}{ Co-morbidity } \\
\hline HIV infection & $6 / 17^{b}(35)$ & $7 / 36^{c}(19)$ & 0.31 \\
\hline Diabetes mellitus & $7(23)$ & $20(34)$ & 0.31 \\
\hline Hemodialysis & $3(10)$ & $2(3)$ & 0.33 \\
\hline Chronic kidney disease & $4(13)$ & $8(14)$ & 1.00 \\
\hline Liver cirrhosis & $1(3)$ & $14(24)$ & 0.02 \\
\hline Chronic lung disease & $1(3)$ & $7(12)$ & 0.26 \\
\hline Steroid user & $11(37)$ & $21(36)$ & 0.92 \\
\hline Solid cancer & $6(20)$ & $5(8)$ & 0.17 \\
\hline Hematologic malignancy & $3(10)$ & $4(7)$ & 0.68 \\
\hline Autoimmune disease & $2(7)$ & $5(8)$ & 1.00 \\
\hline \multicolumn{4}{|l|}{ Disease severity } \\
\hline APACHE-\| score & $12.1 \pm 8.1$ & $13.9 \pm 9.2$ & 0.37 \\
\hline Septic shock & $1(3)$ & $12(20)$ & 0.05 \\
\hline Sepsis & $21(70)$ & $27(46)$ & 0.03 \\
\hline \multicolumn{4}{|l|}{ Laboratory } \\
\hline Leukocyte count $\left(1000 / \mathrm{mm}^{3}\right)$ & $10.46 \pm 7.47$ & $9.94 \pm 6.66$ & 0.74 \\
\hline Hemoglobin (mg/dL) & $11.02 \pm 2.16$ & $11.44 \pm 2.37$ & 0.43 \\
\hline Platelet count $\left(1000 / \mathrm{mm}^{3}\right)$ & $156.90 \pm 107.89$ & $162.44 \pm 98.82$ & 0.81 \\
\hline Neutrophil (\%) & $78.44 \pm 13.40$ & $82.37 \pm 12.14$ & 0.18 \\
\hline Lymphocyte (\%) & $12.84 \pm 9.99$ & $10.23 \pm 8.33$ & 0.20 \\
\hline Serum cryptococcal Ag >1: 512 & $11 / 19^{d}(58)$ & $12 / 31^{e}(39)$ & 0.19 \\
\hline CSF cryptococcal Ag >1:512 & $13 / 20^{f}(65)$ & $16 / 30^{9}(53)$ & 0.41 \\
\hline Specimen from CSF & $15(50)$ & $30(51)$ & 1.00 \\
\hline
\end{tabular}

Azole exposure ${ }^{\mathrm{a}}$ : Patients who received azole therapy (fluconazole, voriconazole, itraconazole, or ketoconazole) for more than $48 \mathrm{~h}$ within 3 months prior to the first episode of invasive cryptococcosis

HIV human immuno-deficiency virus, CSF cerebrospinal fluid, Group 1, patients infected by fluconazole non-susceptible C. neoformans (minimal inhibitory concentrations of fluconazole $\geq 16 \mu \mathrm{g} / \mathrm{ml}$ ); Group 2, patients infected by fluconazole susceptible $C$. neoformans

${ }^{b} 9 ;{ }^{c} 37$ : Numbers of patients with HIV serology test

${ }^{d} 11$; ${ }^{3} 33$ : Numbers of patients with serum cryptococcus $\mathrm{Ag}$

${ }^{f} 11 ;{ }^{9} 32$ : Numbers of patients with CSF cryptococcus Ag

The ability of $C$. neoformans to develop azole resistance is dependent on several mechanisms, including drug target alterations encoded by the gene ERG11, which may be obtained through mutations or by overexpression of the gene encoding, over-expression of efflux pumps, and modulation of stress signaling pathways [30]. Moreover, a pattern of cellular responses to the azoles in C. neoformans has been reported, as well as the term hetero-resistance, which occurs when a single cell gives rise to a progeny with heterogeneous resistance phenotypes, even with a small subset of progeny that are highly resistant to azole [31, 30]. The resistant sub- population can adapt to increasing concentrations of azoles in a stepwise manner [30]. The formation of disomic chromosomes in response to fluconazole stress is closely associated to ERG11 and AFR1, the major transporter of azoles in C. neoformans in both serotypes A and $\mathrm{D}$ [32]. That is an adaptive mechanism in C. neoformans that plays an important role in the failure of fluconazole therapy on cryptococcosis [32].

Pan et al. report that fluconazole has the broadest $(0.125-32 \mu \mathrm{g} / \mathrm{mL})$ and the highest MIC value, and lowest activity $\left(\mathrm{MIC}_{90}=4 \mu \mathrm{g} / \mathrm{mL}\right)$ against $C$. neoformans compared to other azoles like intraconazole, voriconazole, 
Table 2 Stepwise multiple logistic regression analysis of risk factors associated with invasive cryptococcosis due to fluconazole-non-susceptible C. neoformans ${ }^{b}$

\begin{tabular}{llll}
\hline Factors & Comparison & OR $(95 \% \mathrm{Cl})$ & $p$ \\
\hline Admission during 2011-2012 & Yes vs. No & $10.68(2.87-39.74)$ & $<0.001$ \\
Liver cirrhosis & Yes vs. No & $0.17(0.02-1.58)$ & 0.12 \\
Initial present with sepsis $^{\text {a }}$ & Yes vs. No & $1.86(0.62-5.62)$ & 0.27 \\
Azole exposure $^{\text {a }}$ & Yes vs. No & $3.33(0.73-15.25)$ & 0.12
\end{tabular}

Azole exposure ${ }^{a}$ : patients received azole therapy (fluconazole, voriconazole, itraconazole, or ketoconazole) for more than $48 \mathrm{~h}$ within 3 months prior to the first episode of invasive cryptococcosis

$O R$ odds ratio, $\mathrm{Cl}$ confidence interval

${ }^{\text {b }}$ Statistically significant variables in univariate analyses between these categories were entered into multivariate analysis using a logistic regression model. Statistical significance was set at a two-tailed $p<0.05$

posaconazole, and isavuconazole. These new generation triazoles may become an important therapeutic choice to currently used anti-fungals [25].

This study still has several limitations. It is a single center study. Differences in fluconazole-susceptibility rates may exist owing to geographical variations. The retrospective use of patient medical record usually means that some data are missing or misclassified and not all of the patients have been checked for HIV. The number of patients or duration of azole exposure may be underestimated. In addition, drug exposure has not been quantified definitely. These factors may yield more conservative results. This 12-years longitudinal study continuously investigates the susceptibility of $C$. neoformans against fluconazole. In the last two years, there has been increasing fluconazole non-susceptibility. The possibility of a clonal phenomenon associated with fluconazole non-susceptibility is doubtful. However, all patients with invasive cryptococcosis have been diagnosed sporadically for the study period (2001-2012). There is no relationship to admission date and inhabited area among these patients. Further studies with genotyping by pulsed field gel electrophoresis and mating type are needed. Nevertheless, the results here serve to remind clinicians that primary resistance to fluconazole of $C$. neoformans may not be persistently low.

\section{Conclusions}

This study reveals that the fluconazole non-susceptibility of $C$. neoformans has increased in the last two years of this 12-year longitudinal study. Continuous and largescale anti-fungal susceptibility tests for C. neoformans are necessary to confirm this trend.

\section{Abbreviations}

CLSI: Clinical and laboratory standards institutes; MIC: Minimal inhibitory concentration; HIV: Human immune-deficiency virus; IDSA: Infectious disease society of America; CSF: Cerebrospinal fluid; CNS: Central venous system; KCGMH: Kaohsiung Chang Gung Memorial Hospital; AIDS: Acquired immunodeficiency syndrome; APACHE II: Acute physiology and chronic health evaluation II; PaO2: Partial pressure of oxygen in arterial blood;
$\mathrm{PaCO}_{2}$ : Partial pressure of carbon dioxide in arterial blood; PCR: Polymerase chain reaction; DNA: Deoxyribonucleic acid; SDA: Sabouraud dextrose agar; RPMI: Roswell Park Memorial Institute.

\section{Competing interests}

The authors declare that they have no competing interests.

\section{Authors' contributions}

YCC, TYC, JWL, FJC, CCC, CHLee and CHLu participated in the design of this study. FJC, CCC, and CHLee developed the in vitro assay. YCC, CHLee, and CHLu wrote the manuscript. All of the authors read and approved the final manuscript.

\section{Acknowledgements}

This study was supported by grants from Chang Gung Memorial Hospital, Taiwan (CMRPG 8B0671). The authors thank Dr. Gene Alzona Nisperos for editing and reviewing the manuscript for English language considerations.

\section{Author details}

${ }^{1}$ Division of Infectious Diseases, Department of Internal Medicine, Kaohsiung Chang Gung Memorial Hospital, Kaohsiung, Taiwan. ${ }^{2}$ Chang Gung University College of Medicine, 123 Ta-Pei RoadNiao-Sung District, Kaohsiung City 833, Taiwan. ${ }^{3}$ Department of Laboratory Medicine, Kaohsiung Chang Gung Memorial Hospital, Kaohsiung, Taiwan. ${ }^{4}$ Department of Neurology, Kaohsiung Chang Gung Memorial Hospital, 123 Ta-Pei RoadNiao-Sung District, Kaohsiung City 833, Taiwan. ${ }^{5}$ Department of Biological Science, National Sun Yat-Sen University, Kaohsiung, Taiwan.

Received: 28 December 2014 Accepted: 13 July 2015

Published online: 22 July 2015

\section{References}

1. Mitchell TG, Perfect JR. Cryptococcosis in the era of AIDS-100 years after the discovery of Cryptococcus neoformans. Clin Microbiol Rev. 1995;8:515-48.

2. Perfect JR, Dismukes WE, Dromer F, Goldman DL, Graybill JR, Hamill RJ, et al. Clinical practice guidelines for the management of cryptococcal disease: 2010 update by the Infectious Diseases Society of America. Clin Infect Dis. 2010;50:291-322

3. Patel S, Shin GY, Wijewardana I, Vitharana SR, Cormack I, Pakianathan M, et al. The prevalence of cryptococcal antigenemia in newly diagnosed HIV patients in a Southwest London cohort. J Infection. 2013;66:75-9.

4. Berg J, Clancy CJ, Nguyen MH. The hidden danger of primary fluconazole prophylaxis for patients with AIDS. Clin Infect Dis. 1998;26:186-7.

5. Perfect JR, Cox GM. Drug resistance in Cryptococcus neoformans. Drug Resist Updat. 1999:2:259-69.

6. Wang $\mathrm{H}$, Xiao M, Chen SC, Kong F, Sun ZY, Liao K, et al. In vitro susceptibilities of yeast species to fluconazole and voriconazole as determined by the 2010 National China Hospital Invasive Fungal Surveillance Net (CHIF-NET) study. J Clin Microbiol. 2012;50:3952-9.

7. Hsueh PR, Lau YJ, Chuang YC, Wan JH, Huang WK, Shyr JM, et al. Antifungal susceptibilities of clinical isolates of Candida species, Cryptococcus neoformans, and Aspergillus species from Taiwan: surveillance of multi-center antimicrobial resistance in Taiwan program data from 2003. Antimicrob Agents Chemother. 2005;49:512-7.

8. Govender NP, Patel J, van Wyk M, Chiller TM, Lockhart SR, Group for Enteric $\mathrm{R}$, et al. Trends in anti-fungal drug susceptibility of Cryptococcus neoformans isolates obtained through population-based surveillance in South Africa in 2002-2003 and 2007-2008. Antimicrob Agents Chemother. 2011;55:2606-11.

9. Gullo FP, Rossi SA, Sardi Jde C, Teodoro VL, Mendes-Giannini MJ, Fusco-Almeida AM. Cryptococcosis: epidemiology, fungal resistance, and new alternatives for treatment. Eur J Clin Microbiol Infect Dis. 2013;32:1377-91.

10. Lee CH, Chang TY, Liu JW, Chen FJ, Chien CC, Tang YF, et al. Correlation of anti-fungal susceptibility with clinical outcomes in patients with cryptococcal meningitis. BMC Infect Dis. 2012;12:361.

11. Bicanic T, Harrison T, Niepieklo A, Dyakopu N, Meintjes G. Symptomatic relapse of HIV-associated cryptococcal meningitis after initial fluconazole monotherapy: the role of fluconazole resistance and immune reconstitution. Clin Infect Dis. 2006;43:1069-73.

12. Sar B, Monchy D, Vann M, Keo C, Sarthou JL, Buisson Y. Increasing in vitro resistance to fluconazole in Cryptococcus neoformans Cambodian isolates: April 2000 to March 2002. J Antimicrob Chemother. 2004;54:563-5. 
13. Chowdhary A, Randhawa HS, Sundar G, Kathuria S, Prakash A, Khan Z, et al. In vitro anti-fungal susceptibility profiles and genotypes of 308 clinical and environmental isolates of Cryptococcus neoformans var. grubii and Cryptococcus gattii serotype B from north-western India. J Med Microbiol. 2011;60:961-7.

14. Eisenman HC, Casadevall A, McClelland EE. New insights on the pathogenesis of invasive Cryptococcus neoformans infection. Curr Infect Dis Rep. 2007:9:457-64.

15. Knaus WA, Draper EA, Wagner DP, Zimmerman JE. APACHE II: a severity of disease classification system. Crit Care Med. 1985;13:818-29.

16. American College of Chest Physicians/Society of Critical Care Medicine Consensus Conference: definitions for sepsis and organ failure and guidelines for the use of innovative therapies in sepsis. Crit Care Med. 1992;20:864-74

17. Fenn JP, Segal H, Barland B, Denton D, Whisenant J, Chun H, et al. Comparison of updated Vitek Yeast Biochemical Card and API 20C yeast identification systems. J Clin Microbiol. 1994;32:1184-7.

18. Ito-Kuwa S, Nakamura K, Aoki S, Vidotto V. Serotype identification of Cryptococcus neoformans by multiplex PCR. Mycoses. 2007;50:277-81.

19. Aoki FH, Imai T, Tanaka R, Mikami Y, Taguchi H, Nishimura NF, et al. New PCR primer pairs specific for Cryptococcus neoformans serotype A or B prepared on the basis of random amplified polymorphic DNA fingerprint pattern analyses. J Clin Microbiol. 1999;37:315-20.

20. Clinical and Laboratory Standards Institute (CLSI). Reference method for broth dilution anti-fungal susceptibility testing of yeasts; approved standard. 3rd ed. Wayne, PA: CLSI document M27-A3, CLSI; 2008.

21. Dannaoui E, Abdul M, Arpin M, Michel-Nguyen A, Piens MA, Favel A, et al. Results obtained with various anti-fungal susceptibility testing methods do not predict early clinical outcome in patients with cryptococcosis. Antimicrob Agents Chemother. 2006;50:2464-70.

22. Pfaller MA, Diekema DJ, Gibbs DL, Newell VA, Bijie H, Dzierzanowska D, et al. Results from the ARTEMIS DISK Global Anti-fungal Surveillance Study, 1997 to 2007: 10.5-year analysis of susceptibilities of non-candidal yeast species to fluconazole and voriconazole determined by CLSI standardized disk diffusion testing. J Clin Microbiol. 2009;47:117-23.

23. Chen J, Varma A, Diaz MR, Litvintseva AP, Wollenberg KK, Kwon-Chung KJ. Cryptococcus neoformans strains and infection in apparently immunocompetent patients, China. Emerg Infect Dis. 2008;14:755-62.

24. Choi YH, Ngamskulrungroj P, Varma A, Sionov E, Hwang SM, Carriconde F, et al. Prevalence of the VNIc genotype of Cryptococcus neoformans in non-HIV-associated cryptococcosis in the Republic of Korea. FEMS Yeast Res. 2010;10:769-78.

25. Pan W, Khayhan K, Hagen F, Wahyuningsih R, Chakrabarti A, Chowdhary A, et al. Resistance of Asian Cryptococcus neoformans serotype A is confined to few microsatellite genotypes. PLoS One. 2012;7:e32868.

26. Espinel-Ingroff A, Aller Al, Canton E, Castanon-Olivares LR, Chowdhary A, Cordoba S, et al. Cryptococcus neoformans-Cryptococcus gattii species complex: an international study of wild-type susceptibility endpoint distributions and epidemiological cutoff values for fluconazole, itraconazole, posaconazole, and voriconazole. Antimicrob Agents Chemother. 2012;56:5898-906.

27. Tseng HK, Liu CP, Ho MW, Lu PL, Lo HJ, Lin YH, et al. Microbiological, epidemiological, and clinical characteristics and outcomes of patients with cryptococcosis in Taiwan, 1997-2010. PLoS One. 2013;8:e61921.

28. Guinea J, Hagen F, Pelaez T, Boekhout T, Tahoune H, Torres-Narbona M, et al. Antifungal susceptibility, serotyping, and genotyping of clinical Cryptococcus neoformans isolates collected during 18 years in a single institution in Madrid, Spain. Med Mycol. 2010;48:942-8.

29. Cheong JW, McCormack J. Fluconazole resistance in cryptococcal disease: emerging or intrinsic? Med Mycol. 2013;51:261-9.

30. Shapiro RS, Robbins N, Cowen LE. Regulatory circuitry governing fungal development, drug resistance, and disease. Microbiol Mol Biol Rev. 2011;75:213-67.

31. Mondon P, Petter R, Amalfitano G, Luzzati R, Concia E, Polacheck I, et al. Hetero-resistance to fluconazole and voriconazole in Cryptococcus neoformans. Antimicrob Agents Chemother. 1999;43:1856-61.

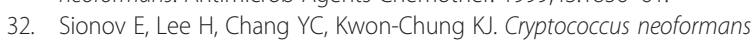
overcomes stress of azole drugs by formation of disomy in specific multiple chromosomes. PLoS Pathog. 2010;6:e1000848.

\section{Submit your next manuscript to BioMed Central and take full advantage of:}

- Convenient online submission

- Thorough peer review

- No space constraints or color figure charges

- Immediate publication on acceptance

- Inclusion in PubMed, CAS, Scopus and Google Scholar

- Research which is freely available for redistribution

Submit your manuscript at www.biomedcentral.com/submit 